\title{
Enhanced antitumor effect of cisplatin in human oral squamous cell carcinoma cells by tumor suppressor GRIM-19
}

\author{
MINGHE LI ${ }^{1,2}$, ZHIHONG LI ${ }^{3}$, JIA LI $^{2}$, LIOU JIN $^{2}$, CHENGXUE JIN ${ }^{2}$, CHENGMIN HAN $^{2}$, XIN JI ${ }^{2}$ and FEI SUN ${ }^{1}$ \\ ${ }^{1}$ Department of Biopharmacy, School of Pharmaceutical Sciences, Jilin University, Changchun, Jilin 130022; \\ ${ }^{2}$ Department of Oral and Maxillofacial Surgery, School of Stomatology Hospital of Jilin University, Changchun, Jilin 130000; \\ ${ }^{3}$ Department of Thoracic Surgery, The First Hospital of Jilin University, Changchun, Jilin 130021, P.R. China
}

Received December 9, 2014; Accepted August 27, 2015

DOI: $10.3892 / \mathrm{mmr} .2015 .4423$

\begin{abstract}
Gene associated with retinoid-interferon-induced mortality 19 (GRIM-19) is a novel candidate tumor suppressor gene located on the human chromosome 19p13.1 region. Our previous study demonstrated that the upregulation of GRIM-19 in human oral squamous cell carcinoma (OSCC) cells significantly inhibited tumor cell growth in vitro and in vivo. In the present study, the combined effects of cationic liposome (LP)-mediated GRIM-19 gene (LP-pGRIM-19) and the low-dose chemotherapeutic drug, cisplatin (CDDP), on tumor cell growth in vitro and in vivo were examined, and the molecular mechanism of their mutual action was investigated by cell proliferation, colony formation, apoptosis, migration, invasion and western blotting assays in vitro, and a node nude tumor model. It was demonstrated that cationic LP-pGRIM-19 gene therapy sensitized the response of breast cancer cells to CDDP, and that LP-pGRIM-19 in combination with CDDP significantly induced apoptosis and inhibited proliferation, colony formation, migration and invasion of the cells, compared with CDDP treatment alone. In addition, systemic treatment with a combination of intravenous injection of LP-pGRIM-19 and intraperitoneal injection of low-dose CDDP into subcutaneous HSC3 human OSCC xenograft mice resulted in a significant inhibition of tumor growth $(\mathrm{P}<0.05)$. Further investigations indicated that the enhancement of CPPP-mediated antitumor effects by GRIM-19 may be associated with the upregulation of phosphorylated p53 and the downregulation of B cell lymphoma-2, cyclin D1, vascular endothelial growth factor, matrix metalloproteinase (MMP)-2 and MMP-9, the proteins of which are involved in the activation of signal transducer and activator of transcription 3 . The results of the present study
\end{abstract}

Correspondence to: Professor Fei Sun, Department of Biopharmacy, School of Pharmaceutical Sciences, Jilin University, 1266 Fujin Road, Changchun, Jilin 130022, P.R. China

E-mail: sunfei408@sina.com

Key words: oral squamous cell carcinoma, gene associated with retinoid-interferon-induced mortality 19, signal transducer and activator of transcription 3 , cisplatin, tumor growth suggested that the combination of GRIM-19 gene therapy with low-dose CPPP-based chemotherapy may be a potent therapeutic strategy for the treatment of OSCC.

\section{Introduction}

Oral cancer, particularly oral squamous cell carcinoma (OSCC), is the most common type of head and neck cancer worldwide, with $\sim 540,000$ new cases annually worldwide $(1,2)$. Despite surgery and chemotherapy being increasingly used to treat OSCC, the 5-year survival rate of OSCC has not improved markedly over previous years, due to late diagnosis, frequent loco-regional recurrences at the primary site and metastasis to neck lymph nodes following treatment $(3,4)$. Therefore, it is imperative to identify novel therapeutics to improve treatment of this disease.

Cisplatin or cis-diammine-dichloroplatinum (II) (CDDP), is a DNA damage-inducing chemotherapeutic drug, and is a chemotherapeutic agent that is widely used in treatment of several types of cancer, including testicular, ovarian, cervical, head and neck, lung, oral and esophageal malignancies (5-7). Despite CDDP being a potent anticancer agent for OSCC, its application is limited due to its side effects, in particular neurotoxicity, which is dose-limiting, and inherent and acquired resistance (8-10). Therefore, novel and customized treatment strategies are required to overcome chemotherapeutic drug resistance and enhance its antitumor activity (11). Targeted therapies, which cause no or minor side effects, may compensate for the limitations of conventional chemotherapies.

It is well known that tumor suppressor genes (TSGs) are important in the pathogenesis of human OSCC and other types of cancer (12-14). The gene associated with retinoid-interferon-induced mortality 19 (GRIM-19) is one of the novel candidate TSGs located on human chromosome 19p13.1, and its overexpression significantly increases cell death (15). Our previous study demonstrated that the forced expression of GRIM-19 in OSCC cells significantly inhibited cell proliferation and colony formation, and induced cell apoptosis in vitro, which effectively suppressed tumor growth in mouse models of human OSCC (16). In addition, it has been demonstrated that the combination of conventional CDDP-based chemotherapy and tumor suppressor gene therapy, including LKB1, p53, NPRL2 and FUS1, may overcome cancer cell resistance 
to chemotherapeutic drugs and enhance therapeutic efficacy in cancer (17-20). However, to the best of our knowledge, no reports are available regarding the combined effects of exogenous expression of GRIM-19 tumor suppressor with any chemotherapeutic drugs, including CDDP, on tumor suppression activity. Therefore, the present study analyzed the potential correlation between the expression of GRIM-19 by cationic liposome (LP)-mediated gene transfer and the enhancement of CDDP sensitivity in human OSCC cell lines. The effects of GRIM-19 on the enhancement of low-dose CDDP-mediated antitumor activity in OSCC cell lines were examined in vitro and in vivo, and the potential molecular mechanisms of the combined treatment of cationic LP-mediated GRIM-19 gene therapy and low-dose CDDP-based chemotherapy were investigated.

\section{Materials and methods}

Cell line, plasmid and chemotherapeutic drug. The HSC3 human oral squamous cell carcinoma cell lines were purchased from the Cell Bank of Type Culture Collection of Chinese Academy of Sciences, Shanghai Institute of Cell Biology, Chinese Academy of Sciences (Shanghai China). The HSC3 cells were cultured in Dulbecco's modified Eagle's medium (DMEM) F-12 medium (Invitrogen Life Technologies, Carlsbad, CA, USA) containing $10 \%$ fetal bovine serum (FBS; Sigma-Aldrich, St. Louis, MO, USA), $1 \%$ fungicide and penicillin/streptomycin (Biochrom, Ltd., Cambridge, UK) at $37^{\circ} \mathrm{C}$ in a humidified atmosphere containing $5 \% \mathrm{CO}_{2} . \mathrm{CDDP}$ was obtained from Sigma-Aldrich. The pVAX1-GRIM-19 (pGRIM-19) plasmid was constructed, as described in our previous study (16). The pVAX1 and pGRIM-19 plasmids were extracted and purified using an Endofree Plasmid Giga kit (Qiagen, Chatsworth, CA, USA) and were solubilized in Endo-free sterilized water for the subsequent experiments. The present study was approved (no. JL2013568B) by the ethics committee of Jilin University (Changchun, China).

Preparation of cationic LPs and plasmid DNA complexes. The cationic LPs, composed of DOTAP/cholesterol (Avanti Polar Lipids, Birmingham, AL, USA) used in the present study were synthesized, as previously described (21). For cell transfection, the pVAX/pVAX-LKB1 plasmid and LPs, diluted in equal volumes of DMEM, were mixed to form DNA:LP complexes (LP-pVAX1 or LP-pGRIM-19), according to their molecular weight ratio (1:6). For the animal experiments, the LPs and plasmid DNA were diluted and mixed in 5\% dextrose, as previous described (17). The average particle size (150-300 nm) of the complexes was selected, according to previous study (17).

Cell viability. To determine CDDP sensitivities, cell viability was performed using a 3-(4,5-dimethylthiazol-2-yl)-2,5-diphenyltetrazolium bromide (MTT) assay. Briefly, the HSC3 cells were seeded $\left(5 \times 10^{3}\right.$ cells/well) onto 96 -well plates and transfected with the different plasmid DNA complexes for $48 \mathrm{~h}$ at $37^{\circ} \mathrm{C}$. Following transfection, DMEM with serial concentrations (1.0-20.0 $\mu \mathrm{M})$ of CDDP were added and incubated for another $48 \mathrm{~h}$ at $37^{\circ} \mathrm{C}$. The cell medium was replaced with $20 \mu \mathrm{l}$ MTT (Sigma-Aldrich) and incubated for another $4 \mathrm{~h}$, followed by the addition of $200 \mu \mathrm{l}$ solubilization solution containing dimethyl sulfoxide (Sigma-Aldrich) into each well. The plates were maintained in a dark room overnight, and the optical density of each sample was measured at a $570 \mathrm{~nm}$ test wavelength using an ELISA multi-well spectrophotometer (SpectraMax ${ }^{\circledR}$ M2/M2e; Molecular Devices Corporation, Sunnyvale, CA, USA). The percentages of viable cells were calculated based on the absorbency of the treated cells relative to that of the untreated cells. The half maximal inhibitory concentration $\left(\mathrm{IC}_{50}\right)$ values of CDDP were also calculated. To evaluate the inhibition on OSCC cell growth by the combined treatment of LP-pGRIM-19 and low-dose CDDP, the half maximal inhibitory concentration $\left(\mathrm{IC}_{50}\right)$ of $\mathrm{CDDP}(10 \mu \mathrm{M})$ was used in all the following experiments in vitro. The HSC3 cells were seeded $\left(5 \times 10^{3}\right.$ cells/well) onto 96-well plates and transfected with either LP-pVAX1 or LP-pGRIM-19, followed by treatment with the $\mathrm{IC}_{50} \mathrm{CDDP}$. The cell viabilities following $48 \mathrm{~h}$ treatment were quantified using an MTT assay, as above described. The visible colonies were then counted under an IX51 inverted microscope (Olympus Corporation, Tokyo, Japan).

Cell colony formation. The HSC 3 cells were seeded into six-well culture plates at $1 \times 10^{4}$ cells/well, and were transfected with either LP-pVAX1 or LP-pGRIM-19, prior to treatment with $\mathrm{IC}_{50} \mathrm{CDDP}$. The cells were incubated at $37^{\circ} \mathrm{C}$ for 10 days, and the medium was replaced every 3 days. The colonies were fixed with ice methanol for $30 \mathrm{~min}$ at room temperature and stained with $2 \%$ Giemsa (Sigma-Aldrich) for $10 \mathrm{~min}$ at room temperature. The visible colonies were then counted.

Cell apoptosis. Flow cytometry was used to detect cell apoptosis. In brief, the HSC3 cells were transfected with either LP-pVAX1 or LP-pGRIM-19, and were then treated with $\mathrm{IC}_{50}$ CDDP for $24 \mathrm{~h}$. Following treatment, the cells were collected and fixed in $4 \%$ paraformaldehyde (Sigma-Aldrich), permeabilized with $70 \%$ ethanol, washed with phosphate-buffered saline (PBS) and stained with propidium iodide (PI; Sigma-Aldrich) solution containing $40 \mu \mathrm{g} / \mathrm{ml}$ PI and $10 \mu \mathrm{g} / \mathrm{ml}$ DNase-free RNase A (Sigma-Aldrich). Apoptosis assays were performed, according to the manufacturer's instructions of the Annexin V-FITC Detection kit (Nanjing KeyGen Biotech Co., Ltd., Nanjing, China). The rates of apoptosis were determined using CellQuest 2.7 software (BD Biosciences, San Jose, CA, USA). In addition, the induction of caspase- $3,-8$ and -9 activity in the HSC 3 cells treated with LP-pGRIM-19 and CDDP were measured using caspase-3, -8 and -9 colorimetric protease assay kits (EMD Millipore, Billerica, MA, USA), according to the manufacturer's instructions. Briefly, the HSC3 cells were seeded into 6-well plates at a density of $4 \times 10^{5}$ cells/well and cultured for $24 \mathrm{~h}$, and collected by centrifugation at $1500 \mathrm{rpm}$ for $5 \mathrm{~min}$ at $4^{\circ} \mathrm{C}$. Caspase-3, -8 , and -9 expression levels were measured in the cell lysates using caspase- $3,-8$, and -9 colorimetric protease assay kits according to the manufacturer's instructions. The optical density was read using a microplate reader (UM313565; Thermo Fisher Scientific Inc., Waltham, MA, USA) at $405 \mathrm{~nm}$.

Invasion and migration assays. Cell invasion assays were performed using a QCM ECMatrix Cell Invasion Assay kit (24-well; $8 \mu \mathrm{m}$; EMD Millipore), according to the 
manufacturer's instructions. Briefly, the HSC3 cells were treated with LP-pGRIM-19 or CDDP for $24 \mathrm{~h}$, and $1 \times 10^{4}$ cells were seeded in the Transwell chamber (BD Biosciences). DMEM supplemented with $20 \%$ FBS was added to the lower chamber as the chemoattractant. Following incubation for $24 \mathrm{~h}$, the non-invading cells in the upper chamber were gently removed using a cotton-tipped swab, and the invading cells in the lower chamber were fixed with methanol and stained with $2 \%$ Giemsa solution. The invasive ability was determined by the number of penetrating cells under a Nikon phase-contrast microscope L150 (Nikon Corporation, Tokyo, Japan) and counted in $>10$ fields of view at x200 magnification in each well.

The in vitro migration assay was similar to the invasion assay described above, however a non-Matrigel-coated 24-well Boyden Chamber ( $8 \mu \mathrm{m}$, Millipore) was used. A total of $2 \times 10^{3}$ cells were added to the Transwell chamber, the incubation duration was $24 \mathrm{~h}$, and the subsequent steps were consistent with the invasion assay. The number of cells migrated were counted by counting the cells in 10 randomly-selected fields per filter, under a Nikon phase-contrast microscope (Nikon Corporation, Tokyo, Japan). Triplicate assays were performed for each group of cells in the invasion and migration assays.

Western blotanalysis. The cells were collected by trypsinization (5\% trypsin; Sigma-Aldrich) and lysed in radioimmunoprecipitation assay lysis buffer (Sigma-Aldrich) with the addition of protease inhibitors (Roche Diagnostics $\mathrm{GmbH}$, Mannheim, Germany) and phosphatase inhibitors (Sigma-Aldrich) for $30 \mathrm{~min}$ on ice. The protein quantity was analyzed using Bradford reagent (Bio-Rad Laboratories, Inc., Hercules, CA, USA). The cell extracts ( $20 \mu \mathrm{g}$ protein) were separated by $10 \%$ SDS-PAGE (Sigma-Aldrich) and transferred onto nitrocellulose membranes (EMD Millipore, Billerica, MA, USA). The membranes were blocked with 5\% dry milk in PBS, and incubated overnight at $4^{\circ} \mathrm{C}$ with the following antibodies: Mouse monoclonal anti-human GRIM-19 (1:2,000; cat. no. sc-365045; Santa Cruz Biotechnology, Inc., Dallas, TX, USA), mouse monoclonal anti-human signal transducer and activator of transcription 3 (Stat3; 1:1,000; cat. no. sc-8019; Bedford, MA, USA), rabbit monoclonal anti-human matrix metalloproteinase (MMP-2; 1:1,000; cat. no. sc-13132; Cell Signaling Technology, Inc., Danvers, MA, USA), mouse monoclonal anti-human MMP-9 (1:2,000; cat. no. sc-21773; Santa Cruz Biotechnology, Inc.), mouse monoclonal anti-human vascular endothelial growth factor (VEGF; 1:2,000; cat. no. sc-53462; Santa Cruz Biotechnology), a mouse monoclonal anti-human phosphorylated (p-)p53 (1:3,000; cat. no. sc-126; Santa Cruz Biotechnology, Inc.), mouse monoclonal anti-human Bcl-2 (1:1,000; cat. no. sc-7382; Santa Cruz Biotechnology, Inc.) and mouse monoclonal anti-human cyclin D1 (1:1,000; cat. no. sc-450; Santa Cruz Biotechnology, Inc.). Mouse monoclonal anti-human $\beta$-actin $(1: 10,000$; cat. no. sc-8432; Santa Cruz Biotechnology, Inc.) was used as a loading control. Following incubation with the primary antibodies, the membranes were washed twice in PBS and incubated with horseradish peroxidase-conjugated goat anti-mouse antibodies (1:5,000; cat. no. sc-2005; Santa Cruz Biotechnology, Inc.) or goat anti-rabbit antibodies (1:5,000; cat. no. sc-2004; Santa Cruz Biotechnology, Inc.) for $2 \mathrm{~h}$ at room temperature. The proteins were detected by the enhanced protein bands, which were visualized using enhanced chemiluminescence reagent (Sigma-Aldrich). The integrated density value (IDV) was analyzed using a computerized image analysis system (Fluor Chen 2.0; Bio-Rad Laboratories, Inc.) and normalized with that of $\beta$-actin.

Tumor growth in vivo. A total of 60 female BALB nude mice aged 4-6 weeks (18-20 g) were purchased from the Institute of Laboratory Animal Science, Jilin University (Changchun, China). A total of $2 \times 10^{6}(100 \mu \mathrm{l})$ HSC3 cells suspended in $100 \mu 1$ PBS were subcutaneously injected into the left abdominal wall, and the size of the resulting tumor was measured daily for 7 days following injection. The tumor volume was calculated as follows: $0.5236 \mathrm{x}^{\text {width }}{ }^{2} \mathrm{x}$ length. At $\sim 20$ days following inoculation of the HSC3 cells, the average tumor volume measured up to $100 \mathrm{~mm}^{3}$. These tumor-bearing nude mice were randomly divided into the following six groups (n=10group): (i) control group; (ii) LP-pVAX1 group; (iii) CDDP group; (iv) LP-pGRIM-19 group; (v) CDDP+LP-pGRIM-19 group; (vi) CDDP+LP-pGRIM-19 group. In the control group, nude mice were injected with $100 \mu 1 \mathrm{PBS}$. Other mice were administered with the LP-pVAX1 or LP-pGRIM-19 complexes by intravenous injection at a dose of $20 \mu \mathrm{g} / \mathrm{mouse}$, and/or low-dose CDDP ( $2 \mathrm{mg} / \mathrm{kg}^{-1} /$ mouse) by intraperitoneal injection once a week for 21 days, respectively. The mice were sacrificed 7 days following the final plasmid injection. Tumor tissue was excised, the volume measured volume and weighed. In addition, splenic tissues were collected and cultured for a splenocyte surveillance investigation using an MTT assay, as described previously (22). Briefly, the splenic tissue samples were collected from the mice, and single-cell spleen suspensions were added to serum-free DMEM by filtering the suspension through a sieve mesh with the aid of a glass homogenizer, in order to exert gentle pressure on the spleen fragments.

Statistical analysis. Data from at least three independent experiments are expressed as the mean \pm standard deviation. Statistical comparison of more than two groups was performed using one-way analysis of variance followed by Tukey's post-hoc test. GraphPad Prism 5.01 software (GraphPad Software, Inc., San Diego, CA, USA) and SPSS ${ }^{\circledR}$ 16.0 (SPSS, Inc., Chicago, IL, USA) for Windows ${ }^{\circledR}$ were used for statistical analyses. $\mathrm{P}<0.05$ was considered to indicate a statistically significant difference.

\section{Results}

Treatment with a combination of LP-pGRIM-19 and low-dose CDDP enhances inhibition of cell proliferation and cell colony formation. To further determine whether the exogenous expression of GRIM-19 sensitizes the response of OSCC cells to CDDP, the present study analyzed the sensitivities of the HSC3 cells to CDDP following treatment with different concentrations of CDDP alone, or combined with LP-pGRIM-19 or LP-pVAX1 transfection, respectively. Compared with CDDP treatment, the $\mathrm{IC}_{50}$ concentrations of CDDP in the HSC3 cells decreased between 7.02 \pm 0.46 and $3.85 \pm 0.41 \mu \mathrm{M}$ when the cells were treated with 
A

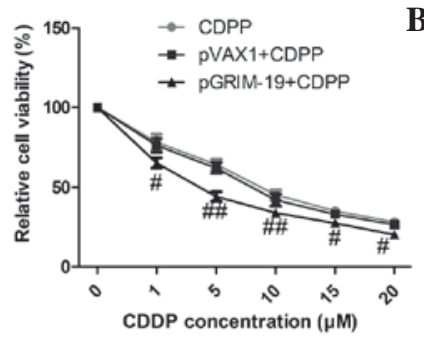

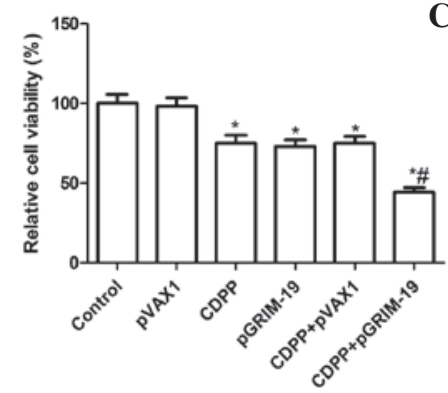

C

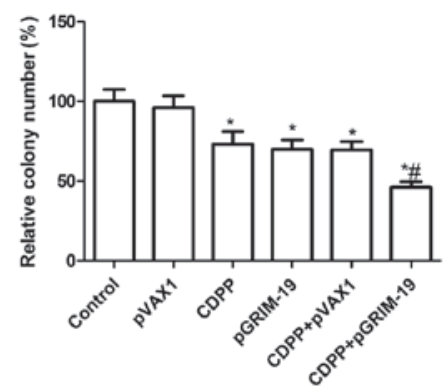

Figure 1. Combined effect of the exogenous expression of GRIM-19 and treatment with CDDP on tumor cell proliferation and clonogenicity in HSC3 cells. (A) HSC3 cells were transfected with LP-pGRIM-19 and treated with various doses of CDDP. Following 48 h treatment, cell viability was analyzed using an MTT assay, and the $\mathrm{IC}_{50}$ values were calculated. (B) HSC3 cells were transfected with LP-pGRIM-19 and then treated with the IC ${ }_{50}$ concentration of CDDP for $48 \mathrm{~h}$. Cell viability was analyzed using an MTT assay. (C) Effects of of LP-pGRIM-19 and low dose CDDP on colony formation of the HSC3 cells were determined following treatment with low-dose CDDP and LP-pGRIM-19, alone and in combination. Data are expressed as the mean \pm standard deviation. ${ }^{*} \mathrm{P}<0.05$, vs. control; ${ }^{\sharp} \mathrm{P}<0.05$, vs. CDDP. GRIM-19, gene associated with retinoid-interferon-induced mortality 19 ; CDDP, cisplatin; LP, liposome; IC ${ }_{50}$, half maximal inhibitory concentration; p, plasmid; MTT, 3-(4,5-dimethylthiazol-2-yl)-2,5-diphenyltetrazolium bromide.

A
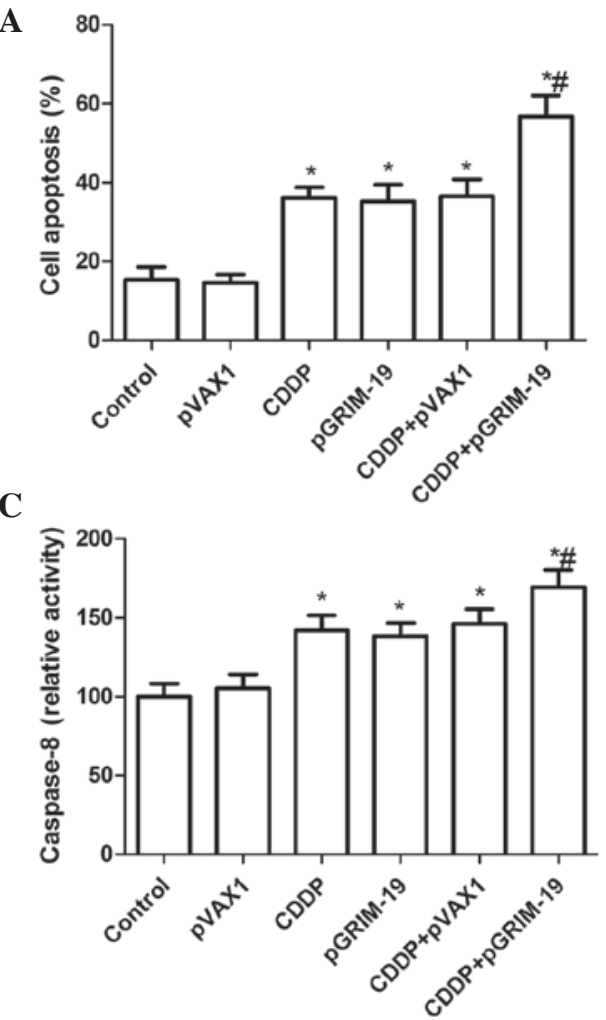

B
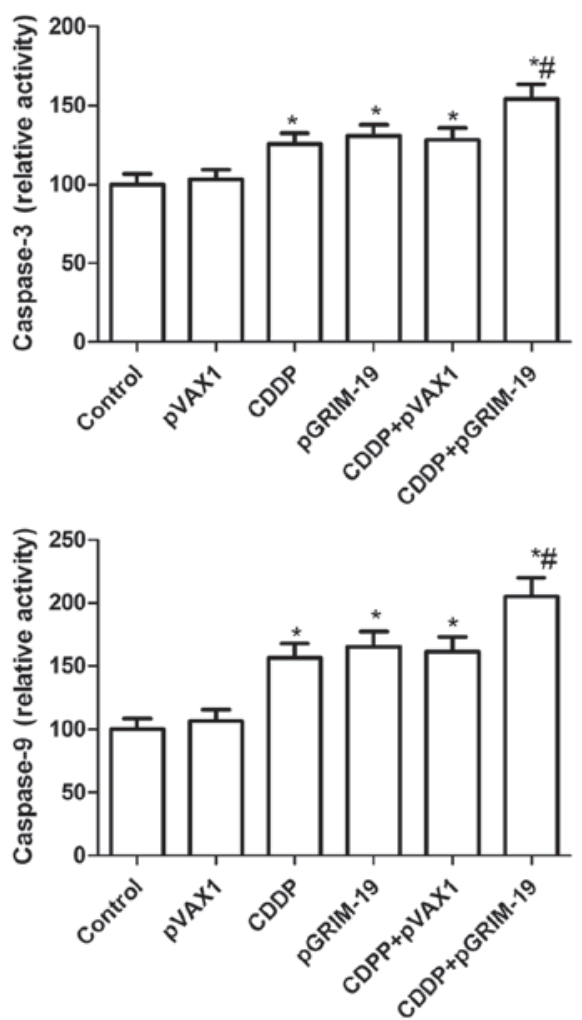

Figure 2. Combined effect of the exogenous expression of GRIM-19 and treatment with CDDP on tumor cell apoptosis in HSC3 cells. (A) Effects of of LPs-pGRIM-19 and low dose CDDP on apoptosis of the HSC3 cells were determined following treatment with low-dose CDDP and LP-pGRIM-19, alone and in combination. Activities of (B) caspase-3 (C) caspase-8 and (D) caspase-9 were determined following treatment with low-dose CDDP and LP-pGRIM-19, alone and in combination. Data are expressed as the mean \pm standard deviation. ${ }^{*} \mathrm{P}<0.05$, vs. control; ${ }^{*} \mathrm{P}<0.05$, vs. CDDP. GRIM-19, gene associated with retinoid-interferon-induced mortality 19; CDDP, cisplatin; LP, liposome; IC50, half maximal inhibitory concentration; $\mathrm{p}$, plasmid.

LP-pGRIM-19+CDDP (Fig. 1A), which suggested that the exogenous expression of GRIM-19 enhanced the sensitivity of the HSC3 cell lines to CDDP. Based on these results the respective $\mathrm{IC}_{50}$ concentrations of CDDP were selected for further treatments in the present study.

To determine whether the exogenous expression of GRIM-19 enhances low-dose CDDP-mediated cell proliferation inhibition, the HSC3 cells were transiently transfected with LP-pGRIM-19 and, following transfection, the cells were treated with low-dose CDDP at the $\mathrm{IC}_{50}$ concentration in The
HSC3 cells. Significantly enhanced inhibition of cell proliferation following treatment with LP-pGRIM-19+low-dose CDDP was observed in the HSC3 cells, compared with treatment with either low-dose CDDP or LP-pGRIM-19 alone $(\mathrm{P}<0.05$; Fig. 1B).

Subsequently, the effects of the combination of LP-pGRIM-19 and low-dose CDDP on colony formation of the HSC 3 cells were analyzed. As shown in Fig. 1C, the combination of LP-pGRIM-19 and low-dose CDDP significantly inhibited the colony formation of the HSC3 cells, compared 

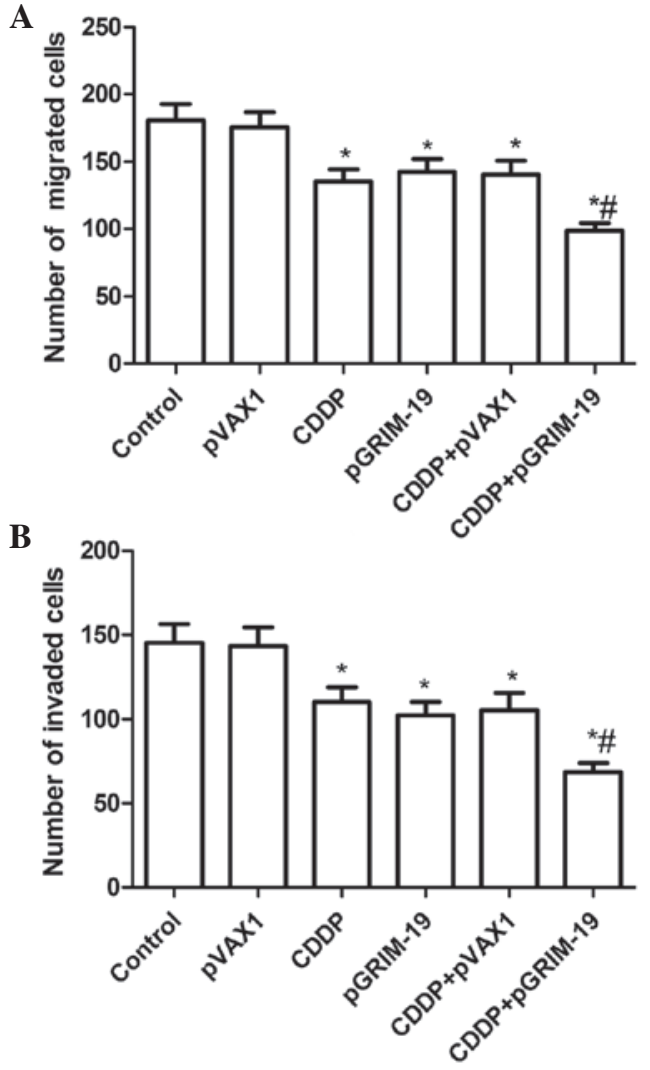

Figure 3. Combined effect of exogenous expression of GRIM-19 and treatment with CDDP on tumor cell migration and invasion in HSC3 cells. (A) Numbers of migrated cells were determined using a Transwell assay (without Matrigel) following treatment with low-dose CDDP and LP-pGRIM-19, alone and in combination. (B) Number of invaded cells were determined using a Transwell matrix penetration assay (with Matrigel) following treatment with low-dose CDDP and LP-pGRIM-19, alone and in combination. Data are expressed as the mean \pm standard deviation. ${ }^{~} \mathrm{P}<0.05$, vs. control; ${ }^{*} \mathrm{P}<0.05$, vs. CDDP. GRIM-19, gene associated with retinoid-interferon-induced mortality 19; CDDP, cisplatin; LP, liposome; IC50, half maximal inhibitory concentration; p, plasmid.

with treatment with either low-dose CDDP or LP-pGRIM-19 alone $(\mathrm{P}<0.05$; Fig. 1C).

Treatment with a combination of LP-pGRIM-19 and low-dose CDDP enhances the induction of HSC3 cell apoptosis. To evaluate whether the exogenous expression of GRIM-19 enhances CDDP-induced cell apoptosis, the HSC3 cells were treated with low-dose CDDP and LP-pGRIM-19 either alone or in combination, and cell apoptosis was detected using flow cytometry. Treatment with LP-pGRIM-19+low-dose CDDP led to a significant increase in apoptosis, compared with either low-dose CDDP or LP-pGRIM-19 treatment alone $(\mathrm{P}<0.05)$, as shown Fig. 2A. In addition, no significance different was observed between the low-dose CDDP- and LP-pGRIM-19-only treatment groups in the induction of OSCC apoptosis.

In order to examine the possible mechanism of the pro-apoptotic effect of the combination of LP-pGRIM-19 and low-dose CDDP, the activities of caspase-3, -8 and-9 were investigated. The results (Fig. 2B-D) indicated that the combination of LP-pGRIM-19 and low-dose CDDP significantly increased the activities of caspase-3, -8 and -9 in the HSC3

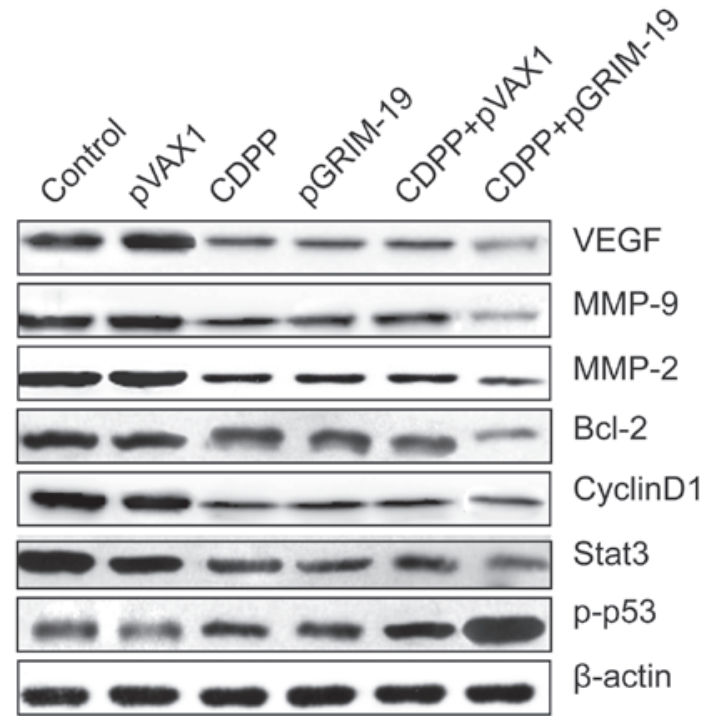

Figure 4. Combined effect of exogenous expression of GRIM-19 and treatment with CDDP on proteins associated with apoptosis and invasion. Protein expression levels of p-p53, Stat3, Bcl-2, cyclin D1, MMP-2, MMP-9 and VEGF were analyzed using western blot assay with specific antibodies following treatment with low-dose CDDP and LP-pGRIM-19, alone and in combination. $\beta$-actin was used as an internal control. GRIM-19, gene associated with retinoid-interferon-induced mortality 19; CDDP, cisplatin; LP, liposome; IC50, half maximal inhibitory concentration; p, plasmid; p-p53, phosphorylated p53; Stat3, signal transducer and activator of transcription 3; Bcl-2, B cell lymphoma 2; MMP, matrix metalloproteinase; VEGF, vascular endothelial growth factor.

cells, relative to either CDDP or LP-pGRIM-19 treatment alone $(\mathrm{P}<0.05)$.

Treatment with a combination of LP-pGRIM-19 and low-dose CDDP enhances the inhibition of cell migration and invasion. The present study aimed to determine whether the combination of LP-pGRIM-19 and low-dose CDDP affected cell vitality, demonstrated by migration or invasion activity. Therefore, cell migration and invasion assays were performed using a Transwell assay. As shown in Fig. 3A, the combination of LP-pGRIM-19 and low-dose CDDP significantly decreased the migration of HSC3 cells, compared with the single LP-pGRIM-19 and CDDP treatment grou[s( $\mathrm{P}<0.05$; Fig. 3A). The ability of this combination to reduce the invasiveness of HSC3 cells was subsequently investigated. The Transwell matrix penetration assay, which was coated with Matrigel revealed that the combination of LP-pGRIM-19 and low-dose CDDP significantly reduced the invasiveness of the HSC3 cells, compared with either CDDP or LP-pGRIM-19 treatment alone $(\mathrm{P}<0.05$; Fig. 3B).

Combination of LP-pGRIM-19 and low-dose CDDP has a synergistic effect on relevant tumor effector molecules in vitro. To elucidate the molecular mechanisms responsible for the induction of synergistic growth inhibition and apoptosis of OSCC cells by LP-pGRIM-19+low-dose CDDP, the expression levels of Bcl-2, cyclinD1, Stat3 and p-p53 were analyzed in cells treated with low-dose CDDP and LP-pGRIM-19 alone and in combination, using western blot analysis. The combination of LP-pGRIM-19 and low-dose CDDP resulted in further upregulation of the protein level of p-p53 and downregulation 
A
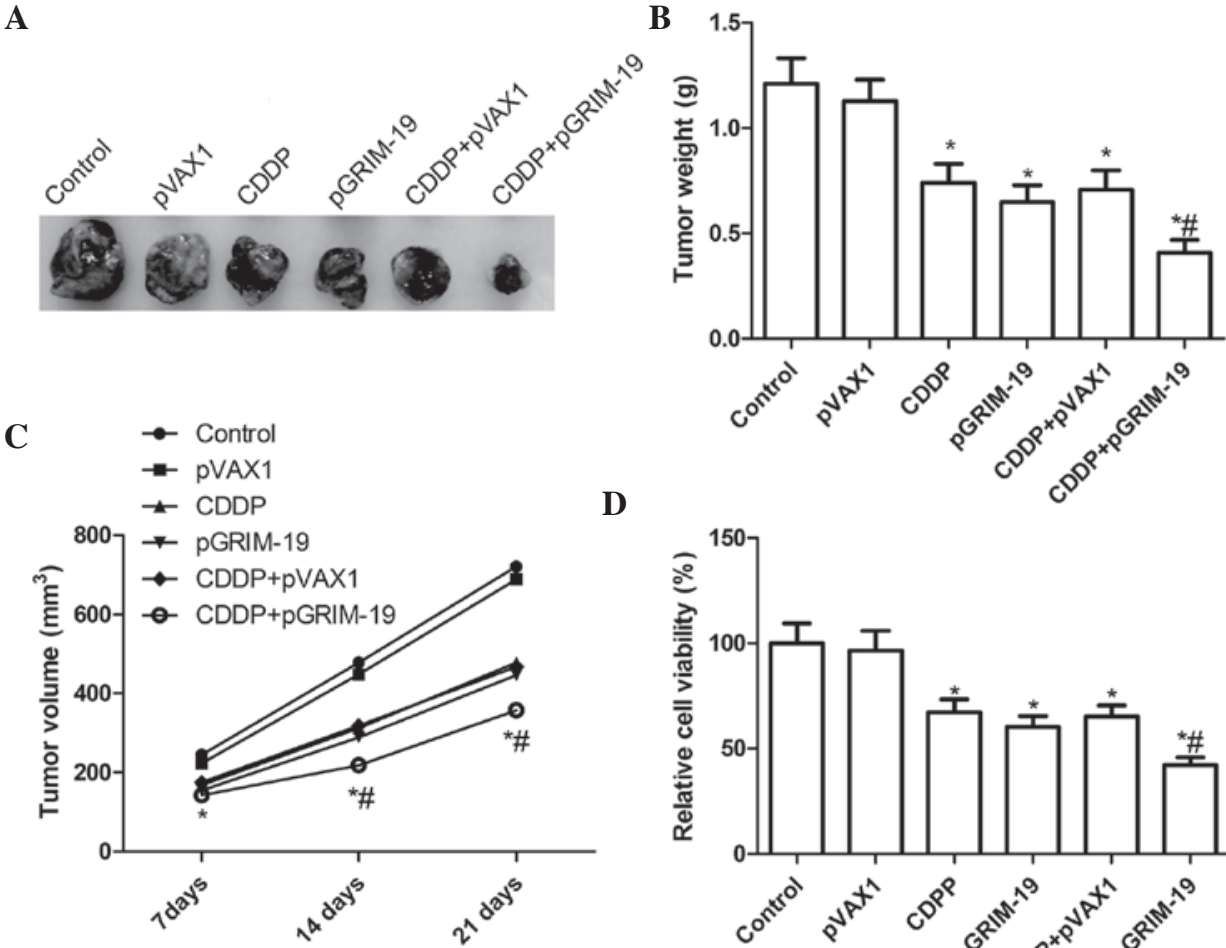

D

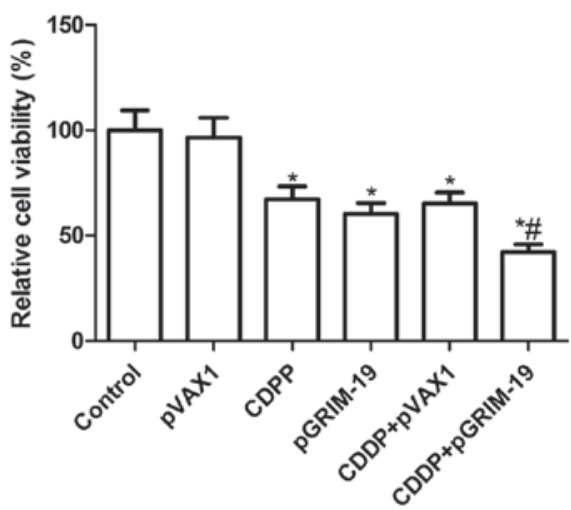

Figure 5. Inhibition of tumor growth in OSCC mouse model by combination treatment with LP-pGRIM-19 and low dose CDDP. A human HSC3 orthotopic OSCC mouse model was used to evaluate the combined effect of systemic administration of LP-pGRIM-19 and low dose CDDP on tumor growth in vivo. (A) Representative images of the tumors in the different treatment groups. (B) Tumor weights in each treatment group were measured on day 21. (C) Tumor volumes in each treatment group were determined at different time-points. (D) Cell viability of splenocytes from mice were determined using a 3-(4,5-dimethylthiazol-2-yl)-2,5-diphenyltetrazolium bromide assay. Data are expressed as the mean \pm standard deviation. ${ }^{*} \mathrm{P}<0.05$, vs. control; " $\mathrm{P}<0.05$, vs. CDDP. GRIM-19, gene associated with retinoid-interferon-induced mortality 19; CDDP, cisplatin; LP, liposome; IC50, half maximal inhibitory concentration; p, plasmid.

of the protein levels of Bcl-2, cyclinD1 and Stat 3 in the HSC3 cells, compared with the LP-pGRIM-19 or low-dose CDDP alone treatment groups (Fig. 4).

To further investigate the potential mechanisms underlying the effects of the combination of LP-pGRIM-19 and low-dose CDDP on cell invasion and migration, relevant effector molecules, including MMP-2, MMP-9 and VEGF were examined using western blot analysis. It was found that the protein expression levels of MMP-2, MMP-9 and VEGF were downregulated in the HSC3 cells treated with of LP-pGRIM-19+low-dose CDDP, compared with treatment with either CDDP or LP-pGRIM-19 alone (Fig. 4)

Treatment with a combination of LP-pGRIM-19 and low-dose $C D D P$ enhances tumor growth inhibition in vivo. To determine whether GRIM-19 gene therapy enhances CDDP-mediated antitumor activity in vivo, the present study evaluated the effects of GRIM-19 combined with low-dose CDDP on tumor regression in female BALB mice bearing HSC 3 tumors. The mice were sacrificed and tumor tissues were removed 21 days following treatment, and the tumor weights were measured. The tumor weights of the of LP-pGRIM-19+low-dose CDDP group were lower than those of the control group and the CDDP-only and LP-pGRIM-19-only groups ( $\mathrm{P}<0.05$; Fig. 5A and $\mathrm{B})$. In addition, the growth in tumor volume in the combination group was significantly slower in the HSC 3 tumor cells, compared with the cispatin-only and LP-pGRIM-19-only groups $(\mathrm{P}<0.05$;
Fig. 5C). Subsequently, MTT assays were used to examine the modulation of splenocyte proliferation and to demonstrate the antitumor activities of this combination in vivo. As shown in Fig. 5D, splenocyte cell proliferation in the combination treatment group decreased significantly, compared with the relative to cispatin-only and LP-pGRIM-19-only groups $(\mathrm{P}<0.05)$. These results suggested that GRIM-19 was responsible for the enhancement of low-dose CDDP-induced antitumor activity in vivo.

\section{Discussion}

Drug resistance is a major cause of treatment failure in patients with OSCC. The combination of conventional CDDP-based chemotherapy and tumor suppressor gene therapy may overcome cancer cell resistance to chemotherapeutic drugs and enhance the therapeutic efficacy in cancer (17-20). However, the role of GRIM-19, a tumor suppressor, in the response to chemotherapy, has not been reported to date, to the best of our knowledge. Therefore, the present study assessed the functional effects of GRIM-19 on the effects of CDDP in OSCC. The results of this investigation demonstrated that exogenously introduced expression of GRIM-19 sensitized the response of OSCC cells to CDDP treatment. Treatment with LP-GRIM-19 in combination with the chemotherapeutic drug, CDDP, markedly inhibited tumor cell proliferation, clonogenicity, migration and invasion, and induced apoptosis in vitro. The present study 
also demonstrated that co-treatment with LP-GRIM-19 and CDDP synergistically suppressed tumor growth in a human OSCC tumor xenograft mouse model. To the best of our knowledge, the present study is the first to report on the combined introduction of the GRIM-19 tumor suppressor and the conventional chemotherapeutic agent, CDDP, in human OSCC cells.

Apoptosis is the primary mechanism causing several malignant cells to die when subjected to chemotherapy or radiotherapy, and it has been demonstrated that mutations in apoptotic pathways and decreased susceptibility to apoptosis are associated with cellular resistance to chemotherapeutic drugs and radiation treatment (23-25). The activation of p53 by CDDP-induced DNA damage has been reported to have various effects on cellular sensitivity towards CDDP (26), whereas downregulation in the expression of $\mathrm{Bcl}-2$ in cancer cells significantly increases the sensitivity of cancer cells to CDDP (27). Our previous study demonstrated that exogenously introduced expression of GRIM-19 in OSCC cells induced cell apoptosis (16). In the present study, the result revealed that the exogenously introduced expression of GRIM-19 sensitized the response of the OSCC cells to CDDP treatment, and that co-treatment with LP-GRIM-19 and CDDP synergistically induced cell apoptosis, increased the activities of caspase-3, -8 and -9 , increased the expression of p-p53 and decreased the expression of Bcl-2. These results suggested that the overexpression of GRIM-19 in CDDP-treated OSCC cells may overcome the cellular resistance and enhance the cellular response to the chemotherapy, by facilitating drug-induced apoptosis, and by upregulating and downregulating the expression levels of p53 and Bcl-, respectively.

The altered expression of Stat 3 has been reported to be key in carcinogenesis by promoting cell proliferation, differentiation and cell cycle progression, as well as inhibiting apoptosis via the incessant induction of pro-growth genes, including cyclin D1, Bcl-2, VEGF and MMP-2 (28-31). The anticancer effects of CDDP can be enhanced by downregulating the expression of Stat3 using Stat3 inhibitors or small interfering RNA $(32,33)$. GRIM-19, a Stat3 inhibitor, has been found to upregulate the expression of GRIM-19 and inhibit Stat3 activation in different types of cancer (28,34-36), due to the binding of GRIM-19 to the Stat 3 gene and inhibiting its transcription. In the present study, exogenously introduced expression of GRIM-19 sensitized the response of OSCC cells to CDDP treatment, and the combination of LP-pGRIM-19 and low-dose CDDP resulted in further upregulation of p-p53, and downregulation of Stat3, Bcl-2, cyclin D1, MMP-2, MMP-9 and VEGF at the ptotein levels in HSC3 cells, compared with either LP-pGRIM-19 or low-dose CDDP treatment alone. These results suggested that the enhanced sensitivity of OSCC cells to CDDP by GRIM-19 may be through inhibition of the Stat 3 signaling pathway.

In conclusion, the present study demonstrated for the first time, to the best of our knowledge, that treatment with LP-pGRIM-19 in combination with a low dose of CDDP markedly inhibited OSCC tumor cell proliferation, clonogenicity, migration and invasion, and induced apoptosis in vitro, as well as suppressing OSCC growth in vivo. The enhanced sensitivity of OSCC cells to CDDP by GRIM-19 was associated with the upregulation of p-p53 and downregulation of Bcl-2, VEGF, MMP-2 and MMP-9 at the protein levels, which are involved in the activation of Stat3. These findings provide novel insight into the molecular mechanisms of GRIM-19-mediated tumor suppression and suggest that the combination of GRIM-19 gene therapy with low-dose CDDP-based chemotherapy may be a potent therapeutic strategy for the treatment of human OSCC.

\section{References}

1. Warnakulasuriya S: Global epidemiology of oral and oropharyngeal cancer. Oral Oncol 45: 309-316, 2009.

2. Siegel R, Naishadham D and Jemal A: Cancer statistics, 2013. CA Cancer J Clin 63: 11-30, 2013.

3. Jerjes W, Upile T, Petrie A, Riskalla A, Hamdoon Z, Vourvachis M, Karavidas K, Jay A, Sandison A, Thomas GJ, et al: Clinicopathological parameters, recurrence, locoregional and distant metastasis in $115 \mathrm{~T} 1-\mathrm{T} 2$ oral squamous cell carcinoma patients. Head Neck Oncol 2: 9, 2010.

4. Forastiere A, Koch W, Trotti A and Sidransky D: Head and neck cancer. New Engl J Med 345: 1890-1900, 2001.

5. O'Dwyer PJ, Stevenson JP and Johnson SW: Clinical pharmacokinetics and administration of established platinum drugs. Drugs 59 (Suppl 4): 19-27, 2000.

6. Baruah H, Barry CG and Bierbach U: Platinum-intercalator conjugates: From DNA-targeted cisplatin derivatives to adenine binding complexes as potential modulators of gene regulation. Curr Top Med Chem 4: 1537-1549, 2004.

7. Shimanishi M, Ogi K, Sogabe Y, Kaneko T, Dehari H, Miyazaki A and Hiratsuka H: Silencing of GLUT-1 inhibits sensitization of oral cancer cells to cisplatin during hypoxia. J Oral Pathol Med 42: 382-388, 2013.

8. Perez RP: Cellular and molecular determinants of cisplatin resistance. Eur J Cancer 34: 1535-1542, 1998.

9. Rabik CA and Dolan ME: Molecular mechanisms of resistance and toxicity associated with platinating agents. Cancer Treat Rev 33: 9-23, 2007.

10. Cohen SM and Lippard SJ: Cisplatin: From DNA damage to cancer chemotherapy. Prog Nucleic Acid Res Mol Biol 67: 93-130, 2001

11. Johnson DH: Evolution of cisplatin-based chemotherapy in non-small cell lung cancer: A historical perspective and the eastern cooperative oncology group experience. Chest 117 (Suppl 1): S133-S137, 2000.

12. Maitra A, Wistuba II, Washington C, Virmani AK, Ashfaq R, Milchgrub S, Gazdar AF and Minna JD: High-resolution chromosome $3 p$ allelotyping of breast carcinomas and precursor lesions demonstrates frequent loss of heterozygosity and a discontinuous pattern of allele loss. Am J Pathol 159: 119-130, 2001.

13. Zabarovsky ER, Lerman MI and Minna JD: Tumor suppressor genes on chromosome $3 p$ involved in the pathogenesis of lung and other cancers. Oncogene 21: 6915-6935, 2002.

14. Lerman MI and Minna JD: The 630-kb lung cancer homozygous deletion region on human chromosome 3 p21.3: Identification and evaluation of the resident candidate tumor suppressor genes. The international lung cancer chromosome $3 \mathrm{p} 21.3$ tumor suppressor gene consortium. Cancer Res 60: 6116-6133, 2000.

15. Angell JE, Lindner DJ, Shapiro PS, Hofmann ER and Kalvakolanu DV: Identification of GRIM-19, a novel cell death-regulatory gene induced by the interferon-beta and retinoic acid combination, using a genetic approach. J Biol Chem 275: 33416-33426, 2000.

16. Li M, Li Z, Liang C, Han C, Huang W and Sun F: Upregulation of GRIM-19 suppresses the growth of oral squamous cell carcinoma in vitro and in vivo. Oncolo Rep 32: 2183-2190, 2014.

17. Ou W, Ye S, Yang W, Wang Y, Ma Q, Yu C, Shi H, Yuan Z, Zhong G, Ren J, et al: Enhanced antitumor effect of cisplatin in human NSCLC cells by tumor suppressor LKB1. Cancer Gene Ther 19: 489-498, 2012

18. Nemunaitis J, Swisher SG, Timmons T, Connors D, Mack M, Doerksen L, Weill D, Wait J, Lawrence DD, Kemp BL, et al: Adenovirus-mediated p53 gene transfer in sequence with cisplatin to tumors of patients with non-small-cell lung cancer. J Clin Oncol 18: 609-622, 2000.

19. Ueda K, Kawashima H, Ohtani S, Deng WG, Ravoori M, Bankson J, Gao B, Girard L, Minna JD, Roth JA, et al: The 3 p21.3 tumor suppressor NPRL2 plays an important role in cisplatin-induced resistance in human non-small-cell lung cancer cells. Cancer Res 66: 9682-9690, 2006. 
20. Deng WG, Wu G, Ueda K, Xu K, Roth JA and Ji L: Enhancement of antitumor activity of cisplatin in human lung cancer cells by tumor suppressor FUS1. Cancer Gene Ther 15: 29-39, 2008.

21. Chen X, Wang X, Wang Y, Yang L, Hu J, Xiao W, Fu A, Cai L, $\mathrm{Li} \mathrm{X}$, Ye X, et al: Improved tumor-targeting drug delivery and therapeutic efficacy by cationic liposome modified with truncated bFGF peptide. J Control Release 145: 17-25, 2010.

22. Zhang H, Li Z and Wang K: Combining sorafenib with celecoxib synergistically inhibits tumor growth of non-small cell lung cancer cells in vitro and in vivo. Oncol Rep 31: 1954-1960, 2014

23. Lowe SW, Ruley HE, Jacks T and Housman DE: p53-dependent apoptosis modulates the cytotoxicity of anticancer agents. Cell 74: 957-967, 1993.

24. Liu JR, Opipari AW, Tan L, Jiang Y, Zhang Y, Tang H and Nuñez G: Dysfunctional apoptosome activation in ovarian cancer: Implications for chemoresistance. Cancer Res 62: 924-931, 2002.

25. Wurstle ML, Zink E, Prehn JH and Rehm M: From computational modelling of the intrinsic apoptosis pathway to a systems-based analysis of chemotherapy resistance: Achievements, perspectives and challenges in systems medicine. Cell Death Dis 5: e1258, 2014.

26. Niedner H, Christen R, Lin X, Kondo A and Howell SB: Identification of genes that mediate sensitivity to cisplatin. Mol Pharmacol 60: 1153-1160, 2001.

27. Park SA, Park HJ, Lee BI, Ahn YH, Kim SU and Choi KS: Bcl-2 blocks cisplatin-induced apoptosis by suppression of ERK-mediated p53 accumulation in B104 cells. Brain Res Mol Brain Res 93: 18-26, 2001.

28. Zhang L, Gao L, Li Y, Lin G, Shao Y, Ji K, Yu H, Hu J, Kalvakolanu DV, Kopecko DJ, et al: Effects of plasmid-based Stat3-specific short hairpin RNA and GRIM-19 on PC-3 M tumor cell growth. Clin Cancer Res 14: 559-568, 2008.
29. Turkson J: STAT proteins as novel targets for cancer drug discovery. Expert Opin Ther Targets 8: 409-422, 2004.

30. Niu G, Wright KL, Huang M, Song L, Haura E, Turkson J, Zhang S, Wang T, Sinibaldi D, Coppola D, et al: Constitutive Stat3 activity up-regulates VEGF expression and tumor angiogenesis. Oncogene 21: 2000-2008, 2002.

31. Xie TX, Wei D, Liu M, Gao AC, Ali-Osman F, Sawaya R and Huang S: Stat 3 activation regulates the expression of matrix metalloproteinase-2 and tumor invasion and metastasis. Oncogene 23: 3550-3560, 2004.

32. Kumar B, Yadav A, Hideg K, Kuppusamy P, Teknos TN and Kumar P: A novel curcumin analog (H-4073) enhances the therapeutic efficacy of cisplatin treatment in head and neck cancer. PloS One 9: e93208, 2014.

33. Han Z, Feng J, Hong Z, Chen L, Li W, Liao S, Wang X, Ji T, Wang S, Ma D, et al: Silencing of the STAT3 signaling pathway reverses the inherent and induced chemoresistance of human ovarian cancer cells. Biochem Biophys Res Commun 435: 188-194, 2013.

34. Zhou Y, Li M, Wei Y, Feng D, Peng C, Weng H, Ma Y, Bao L, Nallar S, Kalakonda S, et al: Down-regulation of GRIM-19 expression is associated with hyperactivation of STAT3-induced gene expression and tumor growth in human cervical cancers. J Interferon Cytokine Res 29: 695-703, 2009.

35. Hao H, Liu J, Liu G, Guan D, Yang Y, Zhang X, Cao X and Liu Q: Depletion of GRIM-19 accelerates hepatocellular carcinoma invasion via inducing EMT and loss of contact inhibition. J Cell Physiol 227: 1212-1219, 2012.

36. Okamoto T, Inozume T, Mitsui H, Kanzaki M, Harada K, Shibagaki N and Shimada S: Upregulation of GRIM-19 in cancer cells suppresses STAT3-mediated signal transduction and cancer growth. Mol Cancer Ther 9: 2333-2343, 2010. 